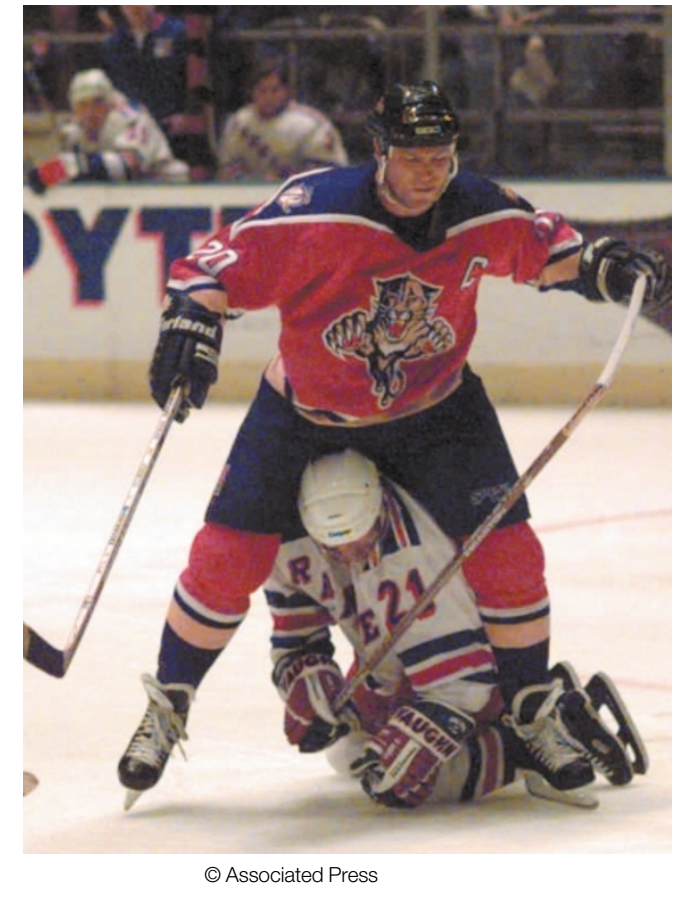

FUNCTIONAL GENOMICS

\section{Comprehensive interference}

A sobering observation that has emerged from all genome sequencing projects so far is that function is unknown for most genes in any organism. So, for post-genomic organisms, such as Caenorhabditis elegans, one important focus of attention is to develop highthroughput approaches for the analysis of gene function. Two recent reports in Nature show how one promising approach involves systematic gene silencing by RNA interference (RNAi).

RNAi refers to the phenomenon whereby exogenously supplied doublestranded RNA (dsRNA) inhibits the function of any endogenous transcripts with the same sequence as the dsRNA. The dsRNA can be supplied by injection or by feeding the worms with Escherichia coli that carry plasmids expressing dsRNA. The method often produces a loss-offunction phenotype, although some genes, tissues or stages of worm development are refractory to RNAi.

The studies by Fraser $e t$ al. and Gönczy et al. both used RNAi to screen comprehensively the predicted genes of one of the six C. elegans chromosomes (I and III, respectively), but the studies differed in their experimental approach. Fraser et al. supplied the dsRNA by feeding, and then screened for a wide range of phenotypic abnormalities during embryonic and post-embryonic development. By contrast, Gönczy et al. supplied the dsRNA by microinjection, and screened embryos using a sensitive microscopic assay for defects in the first two cell divisions of development, as well as a more general assay for later defects. Each study involved around 2,500 genes and identified similar proportions of mutant phenotypes (13.9\% for Fraser et al. and $12 \%$ for Gönczy et al.).

On the face of it, the proportion of genes for which phenotypes were found might sound a bit disappointing, but in sum these two papers provide phenotypic information for more than 400 genes, for which no functional information previously existed. This valuable information is available at WormBase (Fraser et al.) and in a separate public database that includes movies of mutant phenotypes (Gönczy et al.). Among the genes are two homologues of human disease genes for Miller-Dieker lissencephaly and spinal muscular atrophy. And among the surprises is evidence for a new DNA-replication checkpoint that operates early in development. These two studies should provide a significant stimulus to extend RNAi screens to the rest of the genome and to new types of phenotypic screen.

The two groups also looked at the conservation (in the fruitfly and budding yeast) of genes studied in their screens. As might have been expected, the genes associated with phenotypes are more likely to be conserved. In other words, genes involved in basic cellular processes are more likely to generate phenotypes when their function is disrupted. So, the next generation of screens - designed to detect later and more subtle phenotypes - promises to lead to less familiar territory and should yield a rich collection of functional data with which to annotate the worm genome.

Mark Patterson

\section{(2) References and links} ORIGINAL RESEARCH PAPERS Fraser, A. G. et $a$. Functional genomic analysis of $C$. elegans chromosome I by systematic RNA interference. Nature 408, 325-330 (2000) | Gönczy, P. et al. Functional genomic analysis of cell division in C. elegans using RNAi of genes on chromosome Ill. Nature $\mathbf{4 0 8}$, $331-336(2000)$

FURTHER READING RNAi, an online Nature Feature of the Week

WEB SITES Julie Ahringer's lab | Anthony Hyman's lab
GENOMICS

\section{Weed it and reap}

With the publication of its genome sequence, Arabidopsis now ranks among the elite of post-genomic organisms, alongside yeast, worms and flies. Being sessile organisms, it's not surprising that plants have evolved their own molecular peculiarities - Arabidopsis lacks stars of some well-known signal transduction pathways, such as Wnt and Notch, but has many unique protein families, especially among transcription factors. The complete sequence, the quality of which surpasses all other whole-genome sequences published so far, will simplify forward-mutational analyses, although the frequent gene duplications raise the spectre of functional redundancy.

Surveying gene expression patterns provides another avenue for investigating gene function, and a recent microarray analysis of gene expression during the Arabidopsis immune defence response illustrates this point.

"A weed is a plant whose virtues have

not yet been discovered." Ralph Waldo Emerson

When challenged with a pathogen, plants mount both local, transient defence measures and a systemic, long-lasting disease resistance, known as systemic acquired resistance (SAR). One hallmark of the SAR response is the activation of pathogenesis-related (PR) genes that, along with other (unknown) factors, induce a concerted response to an immune challenge.

Maleck et al. examined the changes in gene activity that occur during the induction and maintenance of SAR. Using microarrays, the expression of about 7,000 genes was analysed in plants that had been subjected to fourteen different SARinducing or SAR-repressing treatments. $413 \mathrm{ESTs}$ (1.5\% of the genes in the genome) showed a minimum of 2.5 -fold differential expression in at least two treated samples, which is the conservative criterion used by the authors to select the genes of interest. Plants that were given similar treatments yielded similar gene expression profiles, and these were used to define clusters of genes with co-regulated expression. The cluster that contains the $P R-1$ gene, the most robust marker for SAR, is the most likely to contain other genes involved in SAR, and so was studied in more detail. Backing the assumption that the 31 genes in the $P R-1$ cluster are co-regulated by similar control regions, the cis-elements of 26 genes were found to be highly enriched in W-boxes. These are the binding sites for plant-specific WRKY transcription factors, which the authors speculate might act to repress the SAR response.

This is the first transcriptional profile of the SAR response in Arabidopsis. Follow-up studies should reveal how it's possible to cope when you can't run or you can't hide.

Tanita Casci

(2) References and links

ORIGINAL RESEARCH PAPERS The Arabidopsis Genome Initiative. Analysis of the genome sequence of the flowering plant Arabidopsis thaliana. Nature $\mathbf{4 0 8}$ 796-815 (2000) | Maleck, K. et al. The transcriptome of Arabidopsis thaliana during systemic acquired resistance. Nature Genet. 26, 403-410 (2000)

WEB SITES Jeff Dangl's lab | Experimental data | Arabidopsis Genome Initiative 